\title{
Protective Effect of Total Flavones of Buckwheat Flowers on Carbon Tetrachloride-induced Hepatic Impairment
}

\author{
Jinxiu Chu \\ Department of Pharmacology, North China Coal Medical College \\ 57 South Jian She Road, Tangshan 063000, China \\ E-mail: chujinxiu@126.com \\ Guangmin Li \\ Department of Radiology, Tangshan Womenfolk and Infant Hospital \\ 14 South Jian She Road, Tangshan 063000, China \\ E-mail: chjx000@tom.com \\ Shuying Han (Corresponding author) \\ Department of Pharmacology, North China Coal Medical College \\ 57 South Jian She Road, Tangshan 063000, China \\ E-mail: shuyinghan59@yahoo.com.cn
}

The research is financed by Science Committee of Hebei Province. No. 03276421 (Sponsoring information)

\begin{abstract}
Objective: The protective effect and possible mechanism of total flavones of buckwheat flowers (TFBF) on experimental hepatic impairment in mice were studied. Methods: The hepatic impairment model of mice was induced by injecting carbon tetrachloride $\left(\mathrm{CCl}_{4}\right)$ subcutaneously (sc) every 4 days for 7 times. Meanwhile, mice in the two treatment groups were given TFBF at dosages of $0.04 \mathrm{~g} \cdot \mathrm{kg}^{-1} \cdot \mathrm{d}^{-1}$ and $0.02 \mathrm{~g}^{\circ} \mathrm{kg}^{-1} \cdot \mathrm{d}^{-1}$ respectively through intragastric (ig) injection, and mice in the positive control group were treated with methionine (MET) by contrast. Next the day $\mathrm{CCl}_{4}$ was lastly injected, half the mice were killed. The contents of alanine aminotransferase (ALT) in serum and ALT, superoxidase dismutase (SOD), glutathione (GSH), malonaldehyde (MDA), triglyceride (TG), total cholesterol (TC) in liver tissue, the liver indexes (LI), and the hepato-pathologic changes of the mice were examined. The rest mice were given identical treatment for another 2 weeks. Results: TFBF could inhibit the rising of serum ALT, liver MDA, TG, TC, $\mathrm{LI}$, and the lowering of liver SOD and GSH in $\mathrm{CCl}_{4}$-induced hepatic impairment mice. It could obviously ease the hepato-pathologic damages as well. Conclusion: TFBF could effectively protect the hepatic impairment in $\mathrm{CCl}_{4}$-induced mice.
\end{abstract}

Keywords: Buckwheat, Flavones, Hepatic impairment, Carbon tetrachloride

Buckwheat flowers, which contain abundant flavone compounds such as rutin and quercetin, are blossoms of a plant of polygonaceae named F.esculentum Moench. Studies demonstrated that the flavones of buckwheat possessed many pharmacological activities -- lowering blood glucose and blood lipid, anti-oxidation, improving the hemorheology, regulating vasoactive substances (Han, 2001, pp.694-696; 2003, pp.64-66; 2003, pp477-478; Przybylski, 1998, pp.1595-1601) and so on. But till now no report about its influence on hepatic impairment has been found, so we observed the protective effect of total flavones extracted from buckwheat flowers on $\mathrm{CCl}_{4}$-induced hepatic impairment mice and explored the possible mechanism.

\section{Materials}

\subsection{Animal}

Kunming strain mice of either sex, Grade II, weighing $(21 \pm 2)$ g each, were provided by experimental animal centre of Henan Province, China. Certificate №: 410115. 


\subsection{Medicine and reagent}

TFBF with $90 \%$ purity was extracted in our laboratory from flowers of buckwheat which was planted in Ku Lun Inner Mongolia. Carbon tetrachloride $\left(\mathrm{CCl}_{4}\right)$ (Shanghai) ;Methionine (MET)(Chengdu); Glutamic pyruvic transaminase (ALT), Triglyceride (TG), Total cholesterol (TC), Malonaldehyde (MDA), Superoxidase dismutase (SOD), Glutathione (GSH) reagent (Nanjing).

\subsection{Instruments}

Rotating evaporator (Tianjin); Boehringer Mannheim / Hitachi 7150 Analyzer (Japan); RILI 850 fluorescence emission spectrophotometry equipment (Japan); 721 spectrophotometer (Shanghai); LXJ-II centrifugal equipment (Shanghai); MP200A electronic balance (Shanghai); OLYMPUS VANOXPM-10AD microscopy (Japan).

\section{Methods}

\subsection{Hepatic impairment model making and medicating}

One hundred mice were divided through randomization into 5 groups with 20 in each group, namely, normal group, model group, MET group, high dose TFBF group (HTFBF) and low dose TFBF group (LTFBF). Hepatic impairment model was induced by sc $40 \% \mathrm{CCl}_{4}$-bean oil solution $\left(5 \mathrm{ml} \cdot \mathrm{kg}^{-1}\right)$ every 4 days for 7 times to all of the groups except the normal one which was just treated with bean oil of the same volume. In addition, MET $\left(0.2 \mathrm{~g}^{-\mathrm{kg}^{-1}} \cdot \mathrm{d}^{-1}\right)$, HTFBF $(0.04$ $\left.\mathrm{g} \cdot \mathrm{kg}^{-1} \cdot \mathrm{d}^{-1}\right)$ and LTFBF $\left(0.02 \mathrm{~g} \cdot \mathrm{kg}^{-1} \cdot \mathrm{d}^{-1}\right)$ were given to the relevant groups by ig, while normal and model groups were given water (ig) of the same volume. After a fast of $15 \mathrm{~h}$ from the last injection of $\mathrm{CCl}_{4}, 10$ mice in each group were killed randomly and the targets (I) were detected. The targets (II) of the rest mice were detected after ig medicating for another $2 \mathrm{w}$.

\subsection{Target detection}

After weighing the body weight of each mouse, blood from their eyeballs was taken and centrifuged, and then the content of ALT in serum was determined with the automatic biochemical analyzer. Mice were killed quickly after their blood was taken. Then the livers were taken out, rinsed with cold normal saline, absorbed dry with filter paper, and weighed. The external appearances of the livers were observed with naked eyes and the LI [LI=liver weight $(\mathrm{g}) / \mathrm{body}$ weight $(\mathrm{g}) \times 100 \%$ ] of each mouse was calculated. After that, the left lobe of the liver was halved. One half was made into $10 \%$ even pulp (W/V) with normal saline and was centrifuged. The contents of ALT, SOD, MDA, GSH, and protein in the upper fluid were determined according to the direction. Another half was made into $10 \%$ even pulp (W/V) with methyl alcohol-chloroform (1:1, V/V) and centrifuged. The contents of TG and TC in the upper fluid were determined with the automatic biochemical analyzer. Part of the right lobe of the liver was fixed with $10 \%$ formalin, embedded in paraffin, cut into slices, and stained with H.E. The pathological changes were observed with light microscopy.

\subsection{Statistical analysis}

Statistical analyses were performed with the instat software package. Data were expressed as mean $\pm \operatorname{SD}(\bar{x} \pm s)$ and values of variable were compared with ANOVA $q$ test.

\section{Results}

\subsection{Influence of TFBF on weight and liver indexes in $\mathrm{CCl}_{4}$-induced mice}

From table 1, we can see that the mice in model group lost some weight instead of putting on when treated with $\mathrm{CCl}_{4}$. This meant that $\mathrm{CCl}_{4}$ could inhibit the mice from growing. Different dosages of TFBF could antagonize the inhibition effect of $\mathrm{CCl}_{4}$ on body weight variously. Mice in TFBF groups put on weight more apparently when the irritation of $\mathrm{CCl}_{4}$ was ceased $(P<0.01)$. When $\mathrm{CCl}_{4}$ was injected, $\mathrm{LI}$ of mice in model group were obviously greater than those in normal group. However, they were much smaller in TFBF groups than in model group, especially in HTFBF group. When the irritation of $\mathrm{CCl}_{4}$ was removed, liver indexes of all the groups had little difference.

\subsection{Influence of TFBF on ALT in liver and serum in $\mathrm{CCl}_{4}$-induced mice}

Compared with normal group, the activity of serum ALT in model group was apparently higher $(P<0.01)$. But both dosages of TFBF could lower that markedly $(P<0.01)$, and HTFBF did better. The activity of serum ALT of mice in HTFBF group was almost restored after stopping the injection of $\mathrm{CCl}_{4}$ for $2 \mathrm{w}(P>0.05$, vs normal). It also became lower in model group, but the $P$ value was smaller than 0.01 when compared with normal group. The activity of liver ALT had no significant difference in all of the groups. See also table 2.

\subsection{Influence of TFBF on liver MDA, SOD and GSH in $\mathrm{CCl}_{4}$-induced mice}

$\mathrm{CCl}_{4}$ could increase the amount of liver MDA in mice, but decrease that of SOD and GSH greatly. TFBF could apparently antagonize the rising of MDA and the lowering of SOD and GSH in liver of $\mathrm{CCl}_{4}$-induced mice $(P<0.01)$. Effects on MDA and SOD had no significant difference between the two dosages. Besides, they were approximate to those of MET. As to GSH, HTFBF worked more evidently. See also table 3. 


\subsection{Influence of TFBF on liver TG and TC in $\mathrm{CCl}_{4}$-induced mice}

The contents of liver TG and TC were obviously higher in model group than in normal group $(P<0.01)$, and could be lowered by both dosages of TFBF, particularly HTFBF $(P<0.01)$, which lowered the content of TG more than MET did. See also table 4 .

\subsection{Influence of TFBF on external appearances and pathological changes of liver in $\mathrm{CCl}_{4}$-induced mice}

In normal group, macroscopy of the livers presented dark red with a smooth surface. They were soft and full of flexibility. Microscopically the shape and structure of the liver cells were integral without abnormal changes such as degeneration and necrosis (See also figure 1). In model group, the livers were much friable with gathered volume, a pinkish-grey appearance, and a rough surface. The structure of the liver lobe was destroyed. The liver cells swelled and degenerated with poor-distributed liposome of varying sizes and had obvious vacuolar degeneration. The portal tracts of the liver cells had spotty or focal necrosis and were infiltrated with a lot of inflammatory cells (See also figure 2). In both TFBF groups and MET group, the shape and structure of the livers through macroscopy and microscopy were obviously improved (v.s normal). Degeneration, necrosis, liposome, vascular degeneration, and infiltration of a few inflammatory cells could be found only occasionally (See also figure 3-5). After stopping injecting $\mathrm{CCl}_{4}$ for $2 \mathrm{w}$, the shape and structure of livers of mice in TFBF groups almost tended to be normal and showed regeneration of the cells. In model group, liver cells had some improvement too, but were inferior to those of TFBF groups by comparison.

\section{Discussion}

Hepatism is a kind of disease with a high occurrence rate, which endangers the health of mankind extremely, and is hard to be cured for good. It may be caused by bad diet habits, medicines, and toxicants etc. Hepatic impairment is a complicated process in which many factors participate. $\mathrm{CCl}_{4}$-induced hepatic impairment is a classical hepatoxic model. Its mechanism is that $\mathrm{CCl}_{4}$ can be resolved and activated into free radicals -- $\cdot \mathrm{CCl}_{3}, \mathrm{CCl}_{2},{ }^{\cdot \mathrm{CL}}, \mathrm{OOOCCl}_{3}$ and so on -- by cytochrome $\mathrm{P}_{450}$ of hepatic microbody. Then, these free radicals combine covalently to the macromolecules in hepatic cells, which produces lipid peroxidants, thus damages the constructions and functions of the cell membranes, and dysfunctions the membrane transportation and calcium reserves of the cells, and results in the death of the cells (Nordmann, 1992,29-34) . The results of this experiment showed that $\mathrm{CCl}_{4}$ could obviously increase the amount of serum ALT and liver MDA, TG and TC, but decrease that of liver SOD and GSH in mice. Meanwhile, it could make the LI much greater and cause hepato-pathologic changes including degeneration and necrosis etc. TFBF of both dosages could evidently antagonize the changes above--lower the level of serum ALT, liver MDA, TG, TC and LI, raise that of liver SOD and GSH, and ease hepato-pathologic changes obviously. According to documentary reports ( $\mathrm{Li}, 2003$, 292-294; Wu, 1997, 348-350), flavone compounds had hepatoprotective effect. The mechanism was to inhibit the producing of lipid peroxidants by enhancing the electron transmitting effect between NADP-cytochrome- $\mathrm{P}_{450}$ reductase and $\mathrm{P}_{450}$, reducing the formation of free radicals, and raising the level of radical-scavenging enzyme. The main components of buckwheat flavones are rutin, quercetin, and flavanonol etc. Their hepatoprotective effect is probably that the phenolhydroxyl group in some of the components or their structure can combine to free radicals directly or indirectly and become intermediums of free radicals, thus prevent the chain reactions of free radicals and terminate lipid peroxidation. Studies (Huang, 2000, 589-591) proved that rutin and quercetin etc. could lower the friability but raise the flexibility of capillary membranes to protect them. Document (Liu,2005,315-318) also reported that $\mathrm{NO}$ and $\mathrm{PGI}_{2}$ could vasodilate hepatic vessels, inhibit thrombocyte aggregation, decrease microthrombosis in ischemia areas, improve microcirculation, and lower the activity of serum ALT obviously, so they had protective effect on hepatic cells . Liu (2005, 315-318) et al reported that quercetin had effects of inhibiting protein kinase C and signal transduction. Our research found that TFBF had the effect of regulating vasoactive substances in serum of rats with diabetes mellitus and hyperlipoidemia -- decreasing the amount of ET, AngII \& increasing that of $\mathrm{NO}$ and $\mathrm{PGI}_{2}$-- besides lowering blood glucose , blood lipid and anti-oxidation etc.; therefore, it might be inferred that the hepatoprotective effect of TFBF is relevant to their effects of raising the flexibility but lowering the friability and permeability of hepatic cell membranes, inhibiting $\left[\mathrm{Ca}^{2+}\right]_{\mathrm{i}}$, and regulating vasoactive substances so as to stabilize the membranes and improve microcirculation etc.

In short, TFBF had protective effect on $\mathrm{CCl}_{4}$-induced hepatic impairment. This might be the synthetic result of anti-oxidation, scavenging free radicals, improving microcirculation and lipid metabolism, regulating vasoactive substances, and stabilizing cell membranes etc. The satisfactory mechanism remains to be explored deeply.

Buckwheat is a yearly herb belonging to buckwheat genus of the polygonaceae family. It originates from China, and is widely planted in the north. There are abundant flavone compounds in its flowers and leaves. TFBF function at low specificity and wide scope with no toxicity; in addition, frost comes quite early in the north (Inner Mongolia), and lots of buckwheat can't fruit in autumn so that the local herdsmen use it to feed their livestock. If exploited as medicines and food for health care, waste will be made into treasures. Moreover, we have rich resources. So, buckwheat really has extensive prospects both in exploitation and application. 


\section{References}

Han Shu-Ying, Lv Hua, \& Zhu Li-Sha et al (2001).Effect of total flavones of buckwheat seed on lowering serum lipid,glucose and anti-lipid peroxidation. Chinese Pharmacological Bulletin, 17(6):694-696.

Han Shu-Ying, Liu Shu-Mei, \& Zhu Li-Sha et al (2003).Effect of total flavones of buckwheat flower on vasoactive substances and hemorheology in hyperlipoidemia rat. Northwest Pharmaceutical Journal, 18(2):64-66.

Han Shu-Ying, Zhang Bao-zhong, \& Zhu Li-Sha et al(2003). Cure effect of total flavones of buckwheat flower 0n II diabetic and hyperlipidemia in rats. Chinese Pharmacological Bulletin, 19(4):477-478.

Przybylski R, Lee YC, \& Eskin NAM (1998). Antioxidant and radical-Scavenging activities of Buckwheat seed components, J Am Oil Chem Soc, 75:1595-1601.

Nordmann R. Implication of free radical (1992). Free Rad Bio Med, 12:29-34.

Li Su-Ting, Yang He-Mei, \& Shi Yan-Hua,et al (2003).Protective effects of scutellarriabaicaalensis stem-leaf toal flavonoids on carbon tetrachloride acute hepatic injury in mice. Jounal of CHENG DE Medical College, 20:292-294.

Wu Dong-Fang,Zhou Ben-Hong, \& Luo Shun-De,et al (1997).Effect of Flavonoids of Ginkgo(Ginkgo biloba) on Chemicals Induced Hepatotoxicity in mice.Chinese Traditional and Herbal Drugs, 28:348-350.

Huang He-Sheng,Ma Chuan-Geng, \& Chen Zhi-Geng (2000). Study progress of pharmacology effect of flavone compounds. China Journal of Chinese Materia Medica. 25:589-591.

Bian Cheng, Xu Yong-Hong, \& Li Ding-Guo,et al(2002). Effect of nitrio oxide on experimental hepatic fibrosis in rate. Chinese Pharmacological Bulletin, 18:312-314.

Liu Wen, Liu Xin-Guang, \& Liang Nian-Ci(2002). Effect of sodium quercetin-7-sulfate on recombinant human protein kinase $\mathrm{CK}_{2}$ holoenzyme. Chinese Pharmacological Bulletin, 18:315-318.

Table 1. Effect of TFBF on weight and liver index in injury mice $(\bar{x} \pm s, \mathrm{n}=10)$

\begin{tabular}{|c|c|c|c|c|}
\hline \multirow{2}{*}{ Group } & \multicolumn{2}{|c|}{ Weight/g } & \multicolumn{2}{|c|}{ Liver Index (\%) } \\
\hline & I & II & I & II \\
\hline normal & $+6.6 \pm 1.0$ & $+9.3 \pm 3.2$ & $4.2 \pm 0.5$ & $4.3 \pm 0.4$ \\
\hline model & $-2.5 \pm 0.7$ & $+3.1 \pm 1.5$ & $5.8 \pm 0.9$ & $5.5 \pm 0.7$ \\
\hline HTFBF & $+2.3 \pm 0.6$ & $+7.6 \pm 2.1 * *$ & $4.5 \pm 0.7 *$ & $4.4 \pm 0.8$ \\
\hline LTFBF & $+1.8 \pm 0.6$ & $+7.3 \pm 2.8 * *$ & $4.9 \pm 0.6$ & $4.5 \pm 0.6$ \\
\hline MET & $+1.5 \pm 0.5$ & $+6.4 \pm 1.7 * *$ & $4.8 \pm 0.4 *$ & $4.6 \pm 0.5$ \\
\hline
\end{tabular}

$* P<0.05, \quad * * P<0.01, \quad$ vs model; + increase - decrease

Table 2. Effect of TFBF on ALT in liver and serum in injury mice $(\bar{x} \pm s, \mathrm{n}=10)$

\begin{tabular}{|c|c|c|c|c|}
\hline \multirow{2}{*}{ Group } & \multicolumn{2}{|c|}{ Serum $/ \mathrm{u} \cdot \mathrm{L}^{-1}$} & \multicolumn{2}{|c|}{ Liver/u. $\mathrm{mg}^{-1}$ prot } \\
\hline & I & II & & I \\
\hline normal & $9.4 \pm 2.5$ & $9.2 \pm 2.1$ & $29.6 \pm 4.4$ & $30.7 \pm 3.9$ \\
\hline model & $139.5 \pm 16.2^{\Delta \Delta}$ & $64.6 \pm 7.7 \Delta \Delta$ & $37.8 \pm 4.2$ & $36.4 \pm 3.3$ \\
\hline HTFBF & $42.9 \pm 8.4 * * \Delta \Delta$ & $13.8 \pm 5.9 * *$ & $37.4 \pm 4.7$ & $32.1 \pm 4.2$ \\
\hline LTFBF & $57.2 \pm 8.2 * * \Delta \Delta$ & $21.5 \pm 6.4 * *$ & $34.5 \pm 3.2$ & $29.6 \pm 3.8$ \\
\hline MET & $82.7 \pm 9.7 * * \Delta \Delta$ & $32.1 \pm 5.6^{* * \Delta \Delta}$ & $33.6 \pm 4.1$ & $34.2 \pm 4.9$ \\
\hline
\end{tabular}

$* P<0.05, * * P<0.01, \quad$ vs model ; $\quad \Delta P<0.05, \Delta \Delta P<0.01, \quad$ vs normal 
Table 3. Effect of TFBF on SOD and MDA in mice liver $(\bar{x} \pm s, \mathrm{n}=10)$

\begin{tabular}{|c|c|c|c|c|c|c|}
\hline \multirow{2}{*}{ Group } & \multicolumn{2}{|c|}{$\mathrm{GST} / \mu \cdot \mathrm{mg}^{-1}$ prot } & \multicolumn{2}{|c|}{$\mathrm{MDA} / \mu \mathrm{mol} \cdot \mathrm{g}^{-1}$ prot } & \multicolumn{2}{|c|}{$\mathrm{SOD} / \mu \cdot \mathrm{mg}^{-1}$ prot } \\
\hline & I & II & I & II & I & II \\
\hline normal & $41.6 \pm 3.2$ & $40.3 \pm 2.6$ & $3.8 \pm 0.5$ & $3.6 \pm 0.2$ & $6.7 \pm 0.6$ & $6.2 \pm 0.3$ \\
\hline model & $33.0 \pm 2.8 * * \Delta \Delta$ & $31.6 \pm 3.1 \Delta \Delta$ & $9.1 \pm 0.7$ & $8.5 \pm 0.3^{\Delta \Delta}$ & $3.5 \pm 0.4^{\Delta \Delta}$ & $3.1 \pm 0.4^{\Delta \Delta}$ \\
\hline HTFBF & $38.9 \pm 4.2 * *$ & $39.3 \pm 4.4^{* *}$ & $5.8 \pm 0.6^{* *}$ & $5.5 \pm 0.4 * *$ & $5.2 \pm 0.2 * *$ & $6.4 \pm 0.5 * *$ \\
\hline LTFBF & $35.8 \pm 3.6^{* *}$ & $36.4 \pm 4.2^{* *}$ & $6.2 \pm 0.5^{* *}$ & $5.8 \pm 0.3 * *$ & $4.8 \pm 0.3 * *$ & $5.7 \pm 0.6 * *$ \\
\hline MET & $37.7 \pm 4.3 * *$ & $35.2 \pm 2.3 * *$ & $6.7 \pm 0.6^{* *}$ & $6.1 \pm 0.4^{*}$ & $6.0 \pm 0.4 * *$ & $6.4 \pm 0.4 * *$ \\
\hline
\end{tabular}

$* P<0.05, * * P<0.01, \quad$ vs model ; $\quad{ }^{\circ} P<0.05,{ }^{\Delta} \Delta P<0.01, \quad$ vs normal

Table 4. Effect of TFBF on TG and TC in mice liver $(\bar{x} \pm s, \mathrm{n}=10)$

\begin{tabular}{|c|c|c|c|c|}
\hline \multirow{2}{*}{ Group } & \multicolumn{2}{|c|}{$\mathrm{TG} / \mathrm{mg} \cdot \mathrm{g}^{-1}$ prot } & \multicolumn{2}{|c|}{$\mathrm{TC} / \mathrm{mg} \cdot \mathrm{g}^{-1}$ prot } \\
\hline & I & II & I & II \\
\hline normal & $3.1 \pm 0.2$ & $3.4 \pm 0.33$ & $2.1 \pm 0.3$ & $1.1 \pm 0.2$ \\
\hline model & $6.5 \pm 0.3^{\Delta \Delta}$ & $6.3 \pm 0.3^{\Delta \Delta}$ & $5.7 \pm 0.4^{\Delta \Delta}$ & $2.9 \pm 0.3^{\Delta \Delta}$ \\
\hline HTFBF & $4.3 \pm 0.4 * *$ & $3.7 \pm 0.4 * *$ & $3.3 \pm 0.2 * *$ & $1.4 \pm 0.3 * *$ \\
\hline LTFBF & $4.5 \pm 0.4 * *$ & $3.8 \pm 0.2 * *$ & $3.5 \pm 0.2 * *$ & $1.8 \pm 0.3 * *$ \\
\hline MET & $4.4 \pm 0.4^{*}$ & $3.8 \pm 0.4$ & $3.6 \pm 0.3 * *$ & $1.8 \pm 0.2 * *$ \\
\hline
\end{tabular}

$* P<0.05, * * P<0.01, \quad$ vs model ; $\quad{ }^{*} P<0.05, \Delta \Delta P<0.01$, vs normal

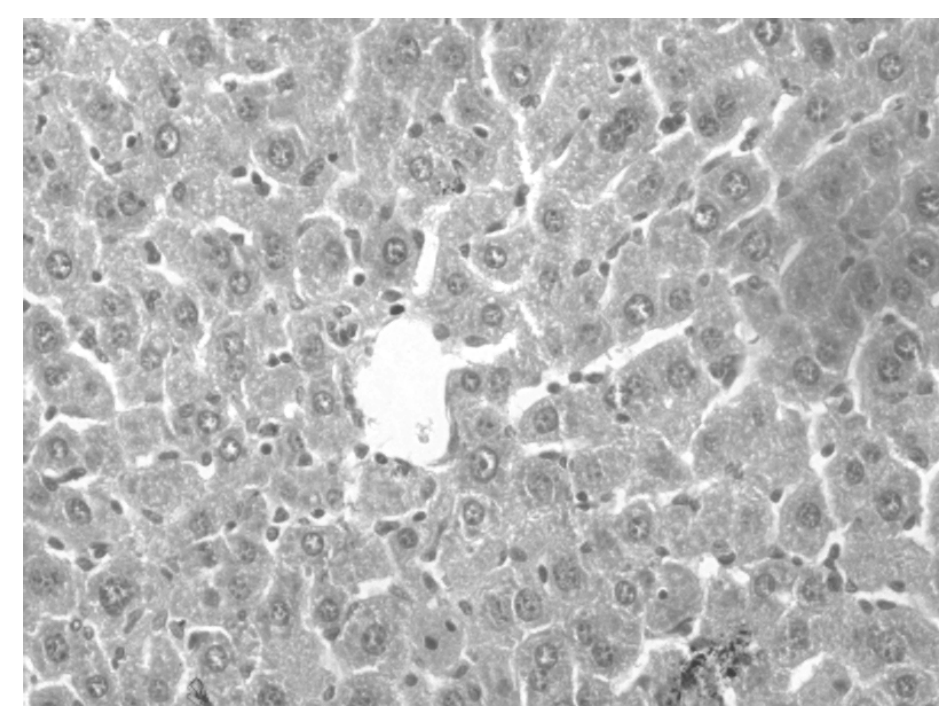

Figure 1. Liver cell of normal group. H.E. $\times 100$ 


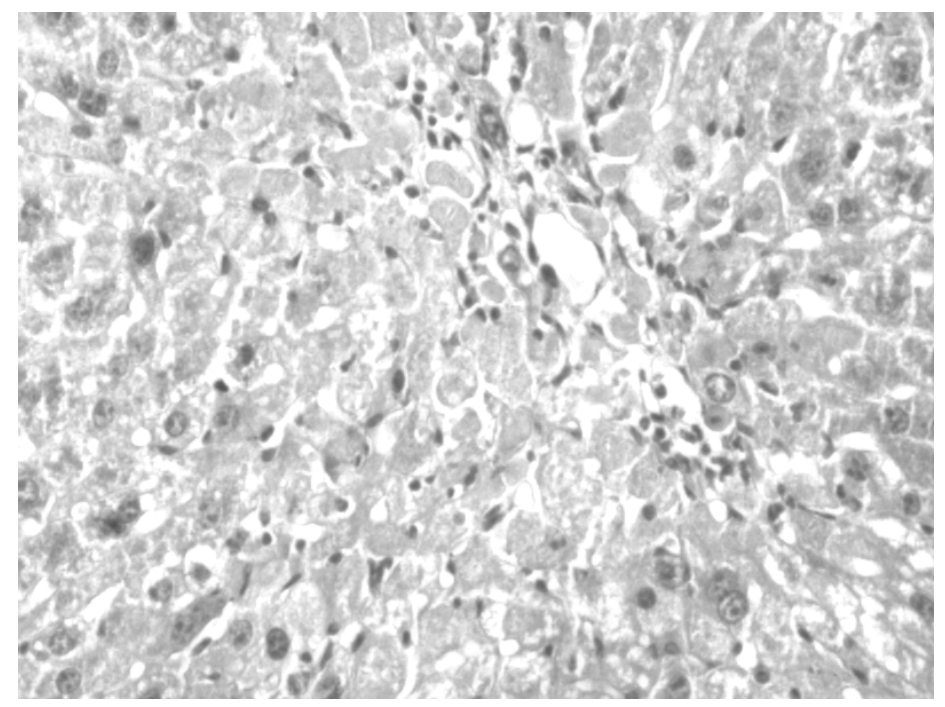

Figure 2. Liver cell of model group. $\quad$ H.E. $\times 100$

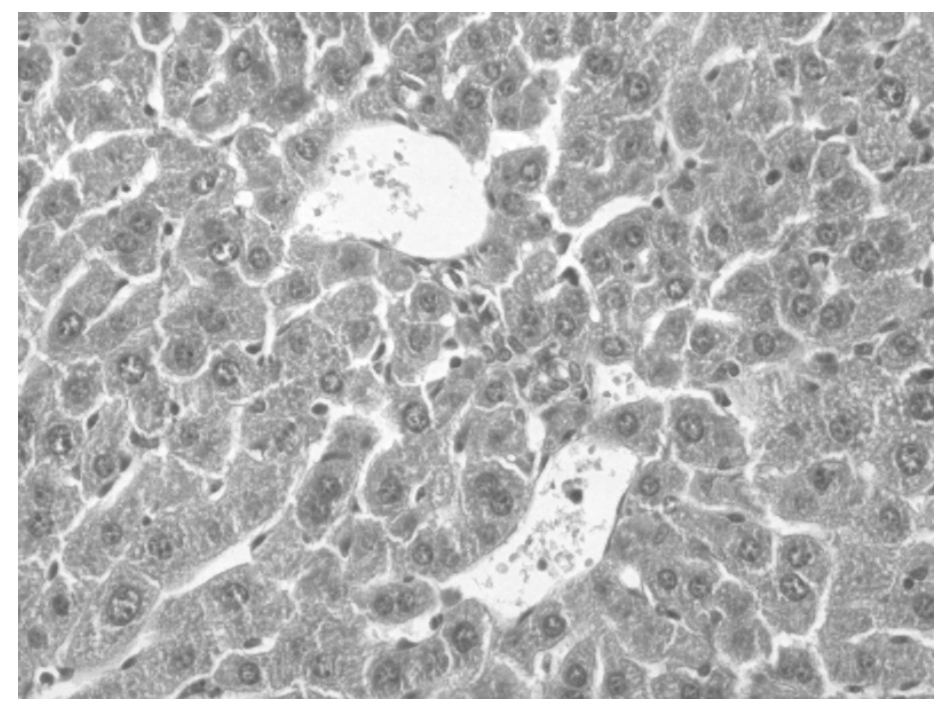

Figure 3. Liver cell of HTFBF group. H.E. $\times 100$

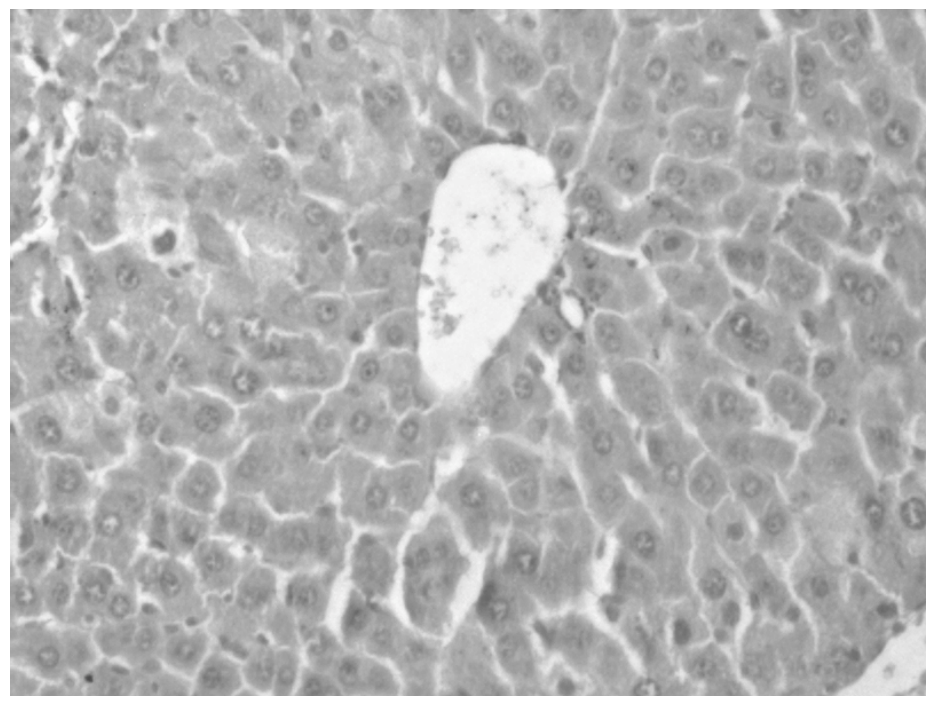

Figure 4. Liver cell of LTFBF group. H.E. $\quad \times 100$ 


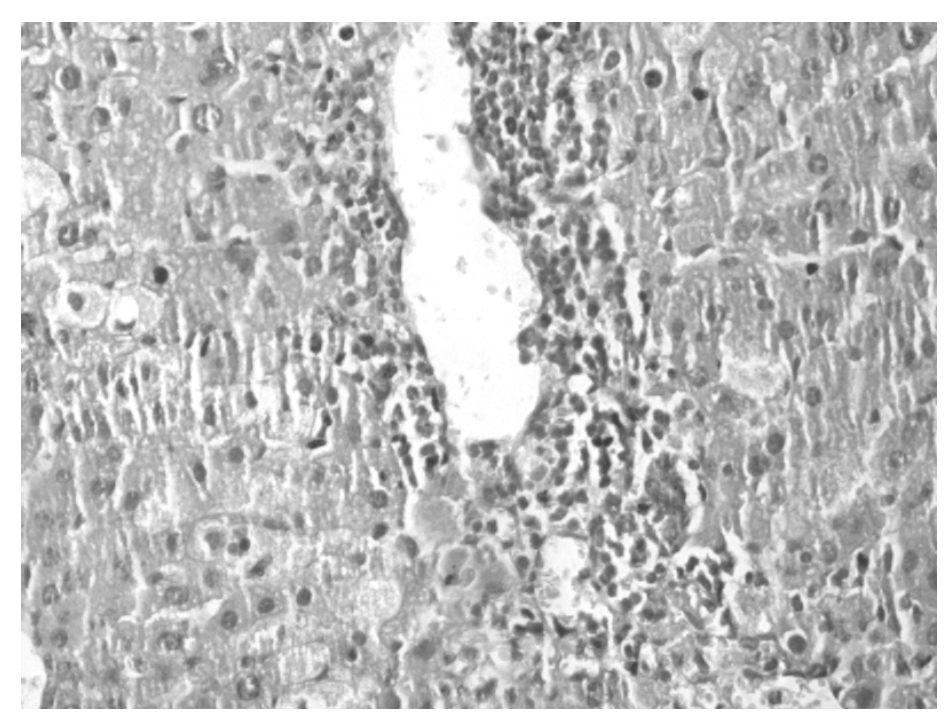

Figure 5. Liver cell of MET group. H.E. $\times 100$ 